\title{
Nocardiosis in central Kerala: A case series
}

$\pi$
0
0
$\frac{1}{4}$
0
$\frac{1}{4}$

Deepthi Karumanchi, Seema Oommen, P. M. Sivan Pillai ${ }^{1}$, Jacinth Angel

Department of Microbiology, Pushpagiri Institute of Medical Sciences and Research Centre, Tiruvalla, ${ }^{1}$ Department of Microbiology, Dr. Somervell Memorial CSI Medical College,

Karakonam, Kerala, India

Address for the Correspondence:

Dr. Seema Oommen, Department of Microbiology, Pushpagiri Institute of Medical Sciences and Research Centre, Tiruvalla - 689 101, Kerala, India.

E-mail: seema.oommen@ gmail.com

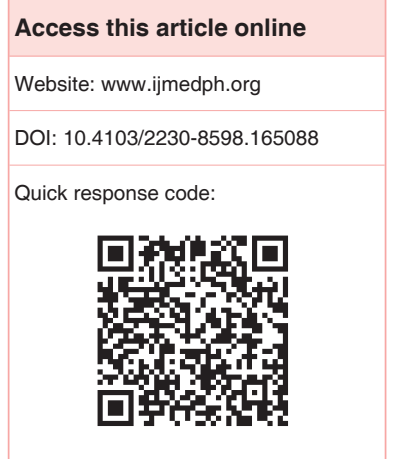

Nocardiosis is an infrequent infection that is often misdiagnosed or under-diagnosed because of its nonspecific clinical picture and infrequent occurrence. Speciation of nocardiae is of clinical importance because of the differences in antibiotic susceptibility patterns and virulence among the species. High level of clinical suspicion and alertness on the part of the laboratory are often required to diagnose these infections. Cases have been reported from other parts of the country, but these are the first series of documented cases from Central Kerala.

Key words: Nocardia, Nocardiosis, Central Kerala

\section{INTRODUCTION}

Nocardiosis caused by Nocardia spp. is an acute, subacute, or chronic infectious disease that occurs in cutaneous, pulmonary, and disseminated forms. ${ }^{[1-3]}$ Primary cutaneous nocardiosis ${ }^{[1-3]}$ usually follows a local traumatic inoculation. It may present as a local cellulitis, abscess or a lymphocutaneous infection (sporotrichoid variant associated with regional lymphadenopathy), or as a subcutaneous infection, e.g., mycetoma. Unlike other forms of nocardiosis, primary cutaneous nocardiosis may present in the immunocompetent as well as in the immunocompromised host. Pulmonary nocardiosis ${ }^{[1-3]}$ presumably follows an exposure from inhalation and presents as an acute, subacute, or chronic pneumonitis with inflammatory endobronchial masses or localized or diffuse pneumonia which may be associated with cavitation, pleural effusion, and empyema. It is commonly encountered in immunocompromised hosts especially with impaired T-cell-mediated immunity. ${ }^{[4]}$ Disseminated nocardiosis ${ }^{[1-3]}$ results from hematogenous spread and are most commonly associated with slowly progressive mass lesions in the brain with or without meningitis.

Nocardia spp. are aerobic actinomycetes that are ubiquitous saprophytes in soil, decomposing organic matter, and fresh or brackish water. Over 50 species of the genus Nocardia have been identified. The taxonomy has been challenging and most likely remains in evolution. ${ }^{[5,6]}$ Most human infections are due to members of the Nocardia asteroides complex. N. asteroides complex was later differentiated by molecular analyses into $N$. abscessus, $N$. brevicatana-paucivorans complex, $N$. nova complex, $N$. transvalensis complex, $N$. farcinica, and $N$. asteroides. ${ }^{[5,6]}$ Speciation of Nocardia is of clinical importance mainly because of varying susceptibility patterns and virulence among the various species. ${ }^{[2,7]}$ By far $N$. farcinica has been documented as the most pathogenic species with resistance to cotrimoxazole, $(80 \%)$ the first line drug used for treatment. ${ }^{[7]}$

\section{CASE REPORTS}

\section{Case 1}

A 48-year-old housewife presented to the surgery outpatient department on 31, December 2012 with complaints of fever, pain and swelling in the right thigh and deltoid region for a period of 1 -week. She had no memory of a history of trauma. The swelling over the deltoid was a $4 \mathrm{~cm} \times 5 \mathrm{~cm}$ lesion while the thigh swelling was around $8 \mathrm{~cm} \times 6 \mathrm{~cm}$, with redness on the skin over the swelling, local rise in temperature and tenderness and of fluid consistency. The patient was a known case of pulmonary hypertension with right heart failure and autoimmune hemolytic anemia for which she was treated earlier with prednisolone and was on currently on maintenance doses. On examination, the patient was afebrile, and other systemic findings were within normal limits. Blood examination revealed hemoglobin of $7.9 \mathrm{~g} / \mathrm{dl}$ and total leukocyte count of $19,000 \mathrm{~cm}$, with thrombocytopenia. A provisional 
diagnosis of an abscess was made, and intravenous ceftriaxone was initiated. The pus was aspirated and sent for culture and sensitivity. The gram stain showed plenty pus cells with few Gram-positive, irregularly stained, branching filamentous bacilli. Modified acid-fast stain showed filamentous acid-fast bacilli resembling Nocardia spp. [Figure 1]. Cotrimoxazole was initiated as therapy upon receipt of the smear report. Chalky white, dry, rough, raised, folded, and irregular colonies suggestive of Nocardia spp. grew on blood agar on the $5^{\text {th }}$ day of incubation at $37^{\circ} \mathrm{C}$ in the candle jar [Figure 2]. The isolate was identified conventionally using a battery of tests as suggested by Kiska et al. ${ }^{[8]}$ as N. nova complex. The patient was treated with linezolid and cotrimoxazole. The lesions resolved, and the patient was discharged on cotrimoxazole with a further review in the outpatient department.

\section{Case 2}

A 39-year-old male patient, farmer by profession, presented with pain and swelling of cystic consistency in the left axilla on 25, July 2013. There was no history of fever or discharge from the swelling. The patient was a known Type II diabetic for the last 25 years and was diagnosed with mantle T-cell lymphoma for which he underwent six cycles of chemotherapy 5 years back, the last cycle of which was completed on 5, April 2013. Hemoglobin was $12 \mathrm{gm} / \mathrm{dl}$, total leukocyte count 24,000 cells $/ \mathrm{mm}^{3}$, with neutrophilia $(82 \%)$. Gram stain revealed thin filamentous, branching bacilli which were acid-fast. He was started on amoxicillin-clavulanate which was supplemented with cotrimoxazole after the smear report. Culture revealed the growth of $N$. brasiliensis. The lesion resolved by 7 days of treatment and the patient was discharged on cotrimoxazole for 6 months.

\section{Case 3}

A 73-year-old housewife, presented on 22, December 2013 in the casualty with fever $102^{\circ} \mathrm{F}$, cough with expectoration and dyspnea for the last 3 days. The patient was a known asthmatic and was on inhaled steroids for the last 3 years. On examination, she had severe

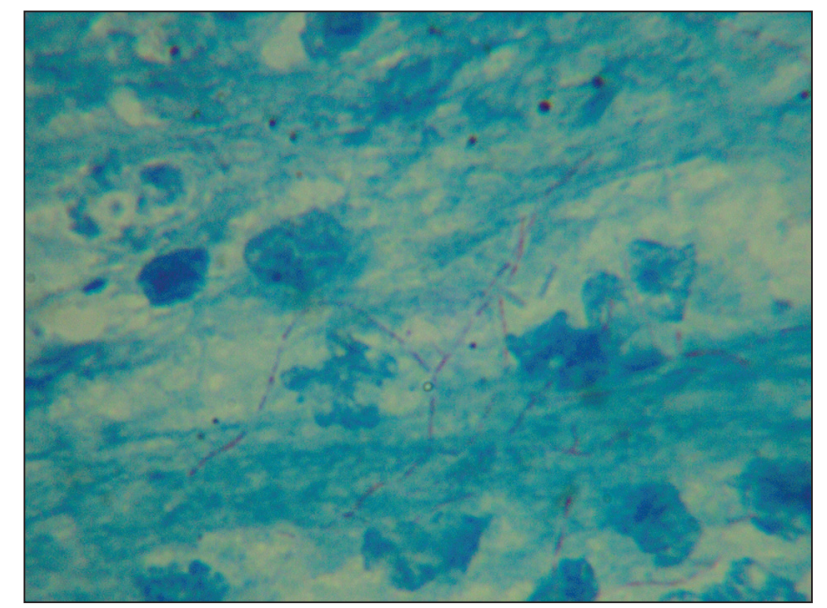

Figure 1: Modified acid-fast stain showing branching filamentous acid-fast bacilli cor pulmonale and auscultation revealed bilateral crepitations and rhonchi. Hemoglobin was $12 \mathrm{gm} / \mathrm{dl}$ and total leukocyte count of 15,000 cells $/ \mathrm{mm}^{3}$ with polymorphs $73 \%$. X-ray chest showed patchy opacity on lower zones on both sides of the lung. Patient was started on azithromycin and ceftriaxone. Gram's stain of sputum revealed Gram-positive bacilli resembling Nocardia spp. Sputum culture revealed the growth of $N$. psendobrasiliensis. The patient was unwilling to initiate cotrimoxazole therapy and was discharged on request.

\section{DISCUSSION}

Depending on the site and the extent of infection combined with underlying host factors, nocardiosis has a variable prognosis, cure rates with appropriate therapy are approximately 100\% in skin and soft-tissue infections, $90 \%$ in pleuropulmonary infections, $63 \%$ in disseminated nocardiosis, and 50\% in patients with brain abscesses. ${ }^{[9]}$ Identification to the species level is important due to the differences in epidemiology, virulence, and antibiotic susceptibilities. ${ }^{[7]}$ Molecular methods such as polymerase chain reaction-restriction fragment length polymorphism and 16S ribosomal DNA sequencing can speciate all medically relevant Nocardia isolates, however, not all laboratories in our country have the capability to performing these tests on a routine basis. Alternatively, biochemical identification as described by Kiska et al. may be used to identify medically relevant Nocardia species in routine laboratories. ${ }^{[8]}$

Cutaneous nocardiosis is acquired via inoculation while the pulmonary lesion is seen following inhalation. Of one of the two cases of cutaneous nocardiosis, one of them was a farmer by profession thus predisposing him to direct inoculation from contaminated soil and water. There was no history of any obvious trauma in the other case. All three cases had obvious T-cell impairment with two of the three patients developing the disease while on long-term steroids. The third patient suffered from T-cell lymphoma and had just completed chemotherapy few months back. This patient was also a Type II diabetic. In a study of assessment of

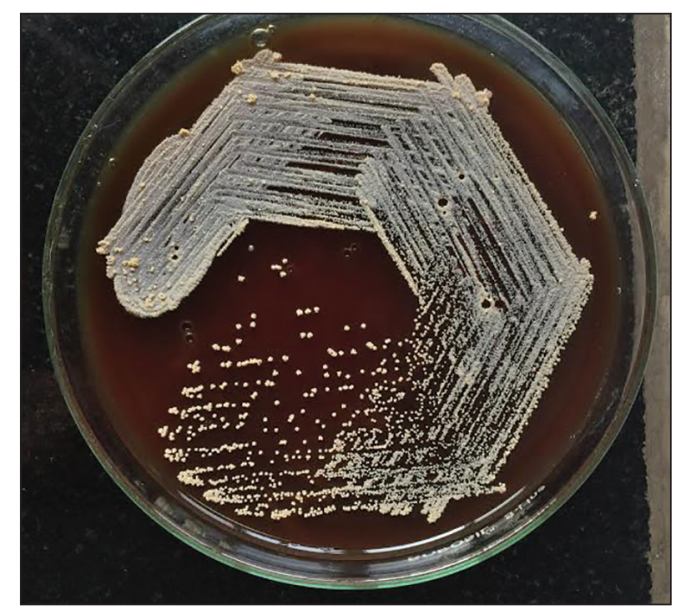

Figure 2: Culture of Nocardia spp. on blood agar 
risk factors by Peleg et al.,$^{[10]}$ high-dose prednisone in the preceding 6 months was significantly associated with Nocardia infection. In a review of 1000 cases of nocardiosis from 1950 to 1991, the most common predisposing factor was organ transplantation followed by malignancies (leukemia and lymphoma) and Acquired Immunodeficiency Syndrome. ${ }^{[4]}$

$N$. asteroides, $N$. farcinica, $N$. nova, $N$. brasiliensis, and $N$. otitidiscaviarum are primary pathogens causing disease in the general population; however, these organisms are more often recognized as opportunistic pathogens in individuals with an underlying condition. ${ }^{[4]} \mathrm{We}$ isolated $N$. nova complex, $N$. brasiliensis and $N$. pseudobrasiliensis in our patients. Traditionally, the first line of therapy for nocardiosis ${ }^{[2]}$ is sulfonamides, especially sulfadiazine because it can penetrate the blood brain barrier. Cotrimoxazole is the standard therapy of choice even though the benefit of trimethoprim component is not clear. Alternatives include imipenem, meropenem, third-generation cephalosporins and amikacin alone or in combination. Combination therapy is recommended for serious infections. Imipenem plus amikacin may be the preferred regimen in sulfonamide allergic individuals. Minocycline, amoxicillin/clavulanate, and linezolid are oral alternatives. Clinical response is often seen in three to 10 days. Protracted specific antimicrobial therapy is the mainstay of medical care for nocardiosis. Therapy is generally recommended for at least 6 months for non-central nervous system (CNS) infections in an immunocompetent patient while immunosuppressed patients and patients with CNS lesions have to be treated for above a year. ${ }^{[2]}$ In patients who require immunosuppressive therapy, antimicrobials against nocardiae is co-administered along with the immune suppressants. In a study conducted by the $\mathrm{CDC}^{[7]}$ on nocardial isolates resistance of $N$. nova complex isolates to amoxyclav was found to be $92 \%$, imipenem 5\%, amikacin 1\%, and cotrimoxazole 53\%. Meanwhile, N. brasiliensis was $11 \%$ resistant to amoxyclav, $83 \%$ to imipenem, $1 \%$ to amikacin, and $20 \%$ to cotrimoxazole. N. pseudobrasiliensis showed $73 \%$ resistance to amoxyclav, $55 \%$ to imipenem, $18 \%$ to amikacin, and $45 \%$ to cotrimoxazole. None of the isolates were resistant to linezolid. The resistance of $N$. farcinica isolates to cotrimoxazole was $80 \%$ necessitating the importance of species identification. Susceptibility to trimethoprim/sulfamethoxazole was performed for our isolates as per the Clinical and Laboratory Standards Institute standards. ${ }^{[1]]}$ All three isolates were sensitive with a minimum inhibitory concentration of $2 / 38 \mu \mathrm{g} / \mathrm{ml}$. All our patients had received empiric antibiotics that were active against Nocardia spp., but the duration of therapy would have been only 5-7 days had it not been for the diagnosis of nocardiosis, making the patient more prone to relapse.
Careful evaluation of all grams smears can help detect this pathogen even if present in low numbers. All suspicious specimens should be subjected to modified Ziehl-Neelsen staining to demonstrate acid-fast bacilli. The importance of microscopy as a diagnostic aid cannot be overemphasized simply due to its ease, speed, and accuracy in diagnosing nocardiosis even before cultures are positive. Nocardia species are usually isolated within three to 5 days. Because nocardiae grow slower than common bacteria, the microbiology laboratory should always be notified when nocardiosis is clinically suspected. This is of particular importance when lower respiratory specimens like sputum are submitted to the laboratory as other colonizing flora may overgrow the pathogen and may lead to premature disposal of cultures. There are no specific clinical signs diagnostic for pulmonary nocardiosis, thus diagnosis of this pathogen requires a great deal of alertness by the clinicians as well as the clinical microbiologist for early detection and institution of effective therapy for better patient outcomes.

\section{REFERENCES}

1. Lerner PI. Nocardiosis. Clin Infect Dis 1996;22:891-903.

2. Wilson JW. Nocardiosis: Updates and clinical overview. Mayo Clin Proc 2012;87:403-7.

3. Corti ME, Villafañe-Fioti MF. Nocardiosis: A review. Int $\mathrm{J}$ Infect Dis 2003;7:243-50.

4. Beaman BL, Beaman L. Nocardia species: Host-parasite relationships. Clin Microbiol Rev 1994;7:213-64.

5. Brown-Elliott BA, Brown JM, Conville PS, Wallace RJ Jr. Clinical and laboratory features of the Nocardia spp. based on current molecular taxonomy. Clin Microbiol Rev 2006;19:259-82.

6. Saubolle MA, Sussland D. Nocardiosis: Review of clinical and laboratory experience. J Clin Microbiol 2003;41:4497-501.

7. Uhde KB, Pathak S, McCullum I Jr, Jannat-Khah DP, Shadomy SV, Dykewicz CA, et al. Antimicrobial-resistant nocardia isolates, United States, 1995-2004. Clin Infect Dis 2010;51:1445-8.

8. Kiska DL, Hicks K, Pettit DJ. Identification of medically relevant Nocardia species with an abbreviated battery of tests. J Clin Microbiol 2002;40:1346-51.

9. Lavalard E, Guillard T, Baumard S, Grillon A, Brasme L, Rodríguez-Nava V, et al. Brain abscess due to Nocardia cyriacigeorgica simulating an ischemic stroke. Ann Biol Clin (Paris) 2013;71:345-8.

10. Peleg AY, Husain S, Qureshi ZA, Silveira FP, Sarumi M, Shutt KA, et al. Risk factors, clinical characteristics, and outcome of Nocardia infection in organ transplant recipients: A matched case-control study. Clin Infect Dis 2007;44:1307-14.

11. Clinical and Laboratory Standards Institute/National Committee for Clinical Laboratory Standards (NCCLS). Susceptibility Testing of Mycobacteria, Nocardiae, and Other Aerobic Actinomycetes: Approved Standard, M24-A. Wayne, PA: NCCLS; 2003.

How to cite this article: Karumanchi D, Oommen S, Sivan Pillai PM, Angel J. Nocardiosis in central Kerala: A case series. Int J Med Public Health 2015;5:384-6.

Source of Support: Nil, Conflicts of Interest: None declared. 